\title{
Design and synthesis of hybrid cyclophanes containing thiophene and indole units via Grignard reaction, Fischer indolization and ring-closing metathesis as key steps
}

\author{
Sambasivarao Kotha ${ }^{*}$, Ajay Kumar Chinnam ${ }^{\ddagger}$ and Mukesh E. Shirbhate ${ }^{\ddagger}$
}

\author{
Full Research Paper \\ Address: \\ Department of Chemistry, Indian Institute of Technology-Bombay, \\ Powai, Mumbai-400 076, India, Fax: 022-2572 7152 \\ Email: \\ Sambasivarao Kotha* - srk@chem.iitb.ac.in \\ * Corresponding author $\ddagger$ Equal contributors \\ Keywords: \\ cyclophane; Grignard reaction; Fischer indolization; ring-closing \\ metathesis
}

\author{
Beilstein J. Org. Chem. 2015, 11, 1514-1519. \\ doi:10.3762/bjoc. 11.165 \\ Received: 01 May 2015 \\ Accepted: 13 August 2015 \\ Published: 31 August 2015 \\ This article is part of the Thematic Series "Progress in metathesis \\ chemistry II". \\ Guest Editor: K. Grela
}

(C) 2015 Kotha et al; licensee Beilstein-Institut.

License and terms: see end of document.

\begin{abstract}
We demonstrate a new synthetic strategy to cyclophanes containing thiophene and indole moieties via Grignard addition, Fischer indolization and ring-closing metathesis as key steps.
\end{abstract}

\section{Introduction}

Modern olefin metathesis catalysts enable a late stage ringclosing step starting with bisolefinic substrates containing polar functional groups [1]. As part of a major program aimed at developing new and intricate strategies to cyclophanes [2-10], we envisioned various building blocks [11] by ring-closing metathesis (RCM) as a key step [12-25]. Cyclophanes containing different heterocyclic systems are difficult to assemble [26-31]. However, we believe that architecturally complex cyclophanes can be accessed by employing a reasonable selection of a synthetic strategy [32]. To enhance the chemical space and also the diversity of cyclophanes the development of powerful and general synthetic methods is highly desirable. Herein, we report a new approach to thiophene- and indole-containing hybrid cyclophane derivatives via Grignard addition, Fischer indolization and RCM as key steps.

\section{Strategy}

The retrosynthetic strategy to the target cyclophane $\mathbf{1}$ containing the thiophene and indole moieties is shown in Figure 1. Here, we conceived thiophene-containing diolefin $\mathbf{3}$ as a possible synthon to assemble the target molecule $\mathbf{1}$ via $\mathbf{2}$. Route A involves an RCM of $\mathbf{3}$ followed by Fischer indolization of $\mathbf{2}$ (Figure 1). Alternatively, Fischer indolization of $\mathbf{3}$ followed by an RCM of diindole 5 can deliver target molecule $\mathbf{1}$ (Route B). The advantages of these approaches are: one can vary the length of the alkene chain during the Grignard addition, and generate 


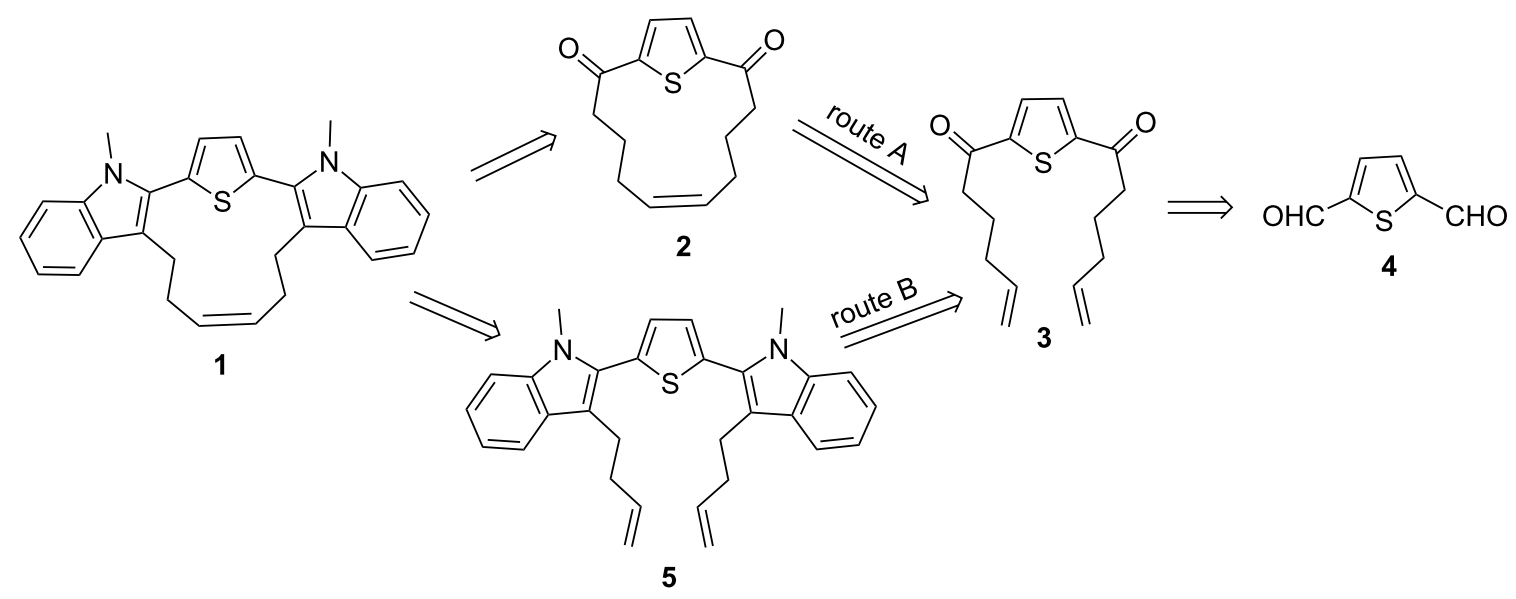

Figure 1: Retrosynthetic approach to hybrid cyclophane derivative 1.

diverse cyclophanes of different ring size. Diverse aromatic rings can be incorporated by altering the aryl hydrazones during the Fischer indolization step. Finally, the additional double bond generated during the RCM sequence can be further manipulated synthetically.

\section{Results and Discussions}

Our synthetic approach to the hybrid cyclophane derivative $\mathbf{1}$ containing thiophene and indole units started with a Grignard addition reaction. In this context, commercially available thiophene-2,5-dicarbaldehyde (4) was reacted with the Grignard reagent [23] derived from 5-bromo-1-pentene to give diol $\mathbf{6}$ as a diastereomeric mixture (Scheme 1). Alternatively, the dialdehyde $\mathbf{4}$ can be prepared by using the Vilsmeier-Haack reaction starting with the thiophene [33]. Later, diol 6 was oxidized with $\mathrm{MnO}_{2}$ [34] to deliver diketone 3. Our attempts to realize the RCM product $\mathbf{2}$ with dione $\mathbf{3}$ via a reaction with Grubbs' catalyst failed to give the expected cyclized product. In most instances, we observed the degradation of the starting material leading to a complex mixture of products as indicated by thinlayer chromatography (TLC). It is known that sulfur can coordinate with the ruthenium catalyst and deactivate the catalytic cycle [35-37]. Therefore, the diolefin did not undergo the RCM sequence.
Next, we explored the alternative option to the target cyclophane 1 involving the bisindolization followed by RCM (Figure 1, Route B). To design aza-polyquinanes, we reported several bisindole derivatives starting with diketones under conditions of a low melting reaction mixture [38-40]. Based on this insight, diketone 3 was subjected to a double Fischer indolization with 1-methyl-1-phenylhydrazine under conditions of a low melting reaction mixture to generate the bisindole derivative $\mathbf{5}$. It is interesting to note that conventional conditions $(\mathrm{AcOH} / \mathrm{HCl})$ for Fischer indolization were not successful with systems related to $\mathbf{3}$. Later, the bisindole derivative $\mathbf{5}$ was subjected to RCM in the presence of Grubbs' $2^{\text {nd }}$ generation catalyst to deliver the desired product $\mathbf{1}$ in good yield (Scheme 2). The sulfur atom present in the bisolefin $\mathbf{3}$ is more accessible for coordination with the Grubbs' catalyst. Whereas in case of the rigid bisindole the sulfur atom is somewhat shielded by the two bulky indole units. Therefore, the bisolefin $\mathbf{5}$ had undergone RCM easily. The structure of compound $\mathbf{1}$ has been assigned on the bases of ${ }^{1} \mathrm{H}$ and ${ }^{13} \mathrm{C}$ NMR spectra. However, the configuration of the double bond present in $\mathbf{1}$ cannot be unambiguously assigned $(\delta=5.63, \mathrm{t}, J=5.40 \mathrm{~Hz}$, $2 \mathrm{H})$. The stereochemistry of the double bond was assigned based on single crystal X-ray diffraction studies and it was found to be the cis (Figure 2) [41].<smiles>C=CCCCCC(=O)c1ccc(C(=O)CCCC(=O)c2ccc([C@@H](O)CCCC=C)s2)s1</smiles> 


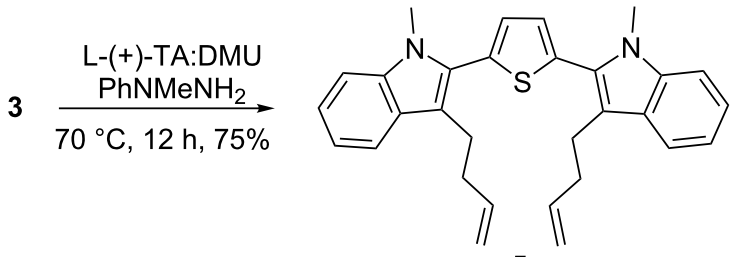

5

\section{$\underset{\text { it, } 24 \mathrm{~h}, 90 \%}{\stackrel{\mathrm{Cll}}{\mathrm{CH}} \mathrm{Cl}_{2}}$}<smiles>C/C=C\CCc1c(CC/C=C\CCC2=C(c3ccc(-c4cccn4C)s3)c3ccccc32)n(C)c2ccccc12</smiles>

1

Scheme 2: Synthesis of hybrid cyclophane 1.

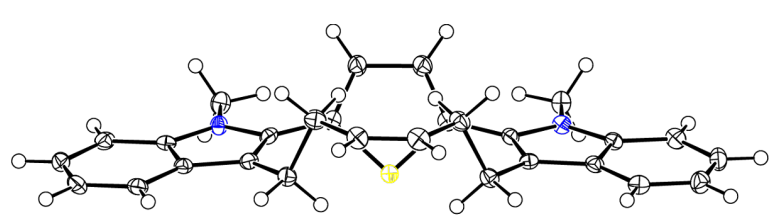

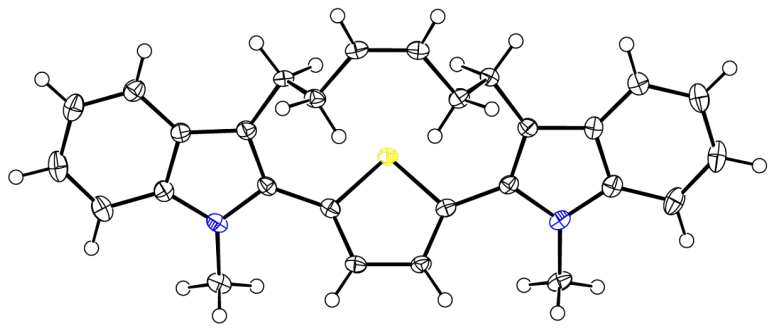

Figure 2: The molecular crystal structure of 1 with $50 \%$ probability [41].
Having, demonstrated the RCM step, next, we attempted to expand this strategy. In this regard a synthesis of a higher analogue containing seven carbon alkenyl chains was undertaken. To achieve this goal, thiophene dicarbaldehyde $\mathbf{4}$ was subjected to a Grignard addition reaction with hexenylmagnesium bromide which gave diol $\mathbf{6 a}$ as a mixture of diastereomers. Further, the diol was subjected to an oxidation step in the presence of $\mathrm{MnO}_{2}$ to generate dione 3a. Later, RCM was attempted with various Grubbs' catalysts. However, the RCM product 2a was not realized (Scheme 3 ). Under similar reaction conditions, dione 3a was converted into the bisindole derivative $\mathbf{5 a}$ by using the Fischer indolization and subsequently an RCM protocol to convert 5a to the cyclized product 1a (Scheme 4). Based on the structure of compound $\mathbf{1}$, here also we anticipate the double bond stereochemistry as "cis".

\section{Conclusion}

We have developed a simple synthetic strategy to hybrid cyclophane derivatives $\mathbf{1}$ and 1a containing thiophene and indole

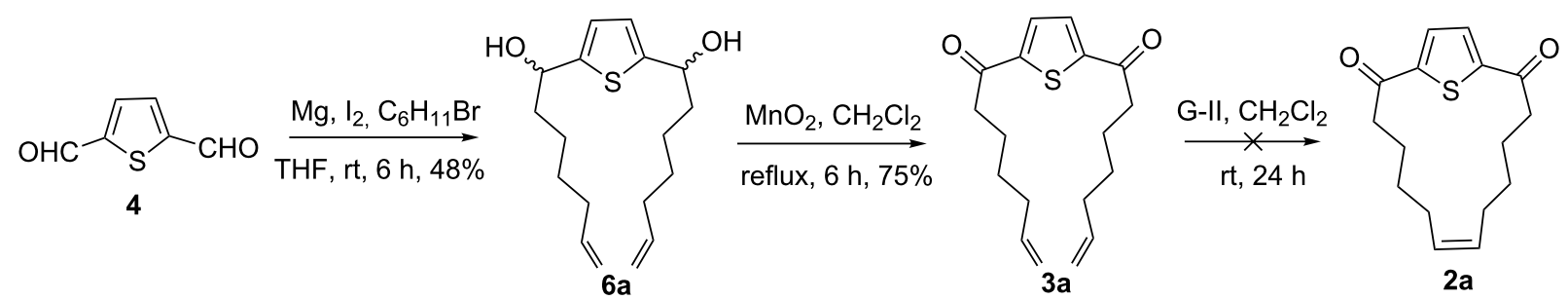

Scheme 3: Attempted synthesis of thiophenophane derivative 2a.

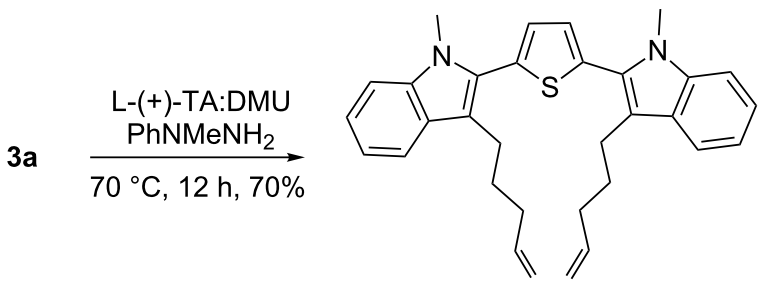

$5 \mathbf{a}$

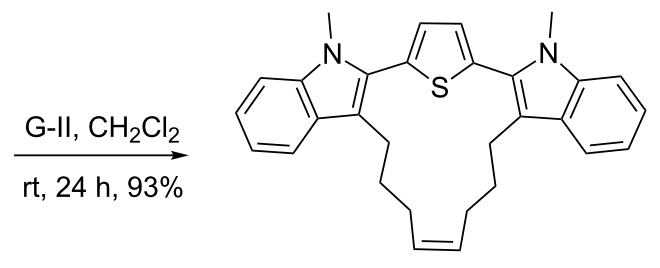

$1 \mathrm{a}$ 
moieties. Simple dialkene-containing thiophene derivative 3a failed to deliver the RCM product. However, the sterically congested bisindole systems 5 and 5a undergo RCM easily. Here, the bulky indole moieties shield the sulfur atom and prevent its coordination with the catalyst. In essence, the power of this synthetic strategy has been harnessed to realize complex cyclophanes starting with simple synthons.

\section{Experimental}

Analytical TLC was performed on $(10 \times 5 \mathrm{~cm})$ glass plate coated with silica gel $\mathrm{GF}_{254}$ (containing $13 \% \mathrm{CaSO}_{4}$ as a binder). Visualization of the spots on the TLC plate was achieved by exposure to UV light and/or $\mathrm{I}_{2}$ vapor. Column chromatography was performed using silica gel (100-200 mesh) and the column was usually eluted with an ethyl acetate/petroleum ether mixture (bp $60-80{ }^{\circ} \mathrm{C}$ ). Melting points were recorded on a Büchi apparatus. ${ }^{1} \mathrm{H}$ NMR and ${ }^{13} \mathrm{C}$ NMR spectral data were recorded on Bruker 400 and $500 \mathrm{MHz}$ spectrometers using TMS as an internal standard and $\mathrm{CDCl}_{3}$ as solvent. The coupling constants $(J)$ are given in hertz $(\mathrm{Hz})$. Chemical shifts are expressed in parts per million (ppm) downfield from internal reference, tetramethylsilane. The standard abbreviation s, d, t, q, m, dd and td, refer to singlet, doublet, triplet, quartet, multiplet, doublet of doublet, and triplet of the doublet, respectively. Mass spectral data were recorded on a Q-TOF micromass spectrometer. For the preparation of anhydrous THF, initially it was passed through a column of activated alumina. Later, it was refluxed over and distilled from $\mathrm{P}_{2} \mathrm{O}_{5}$ and stored over sodium wire. Other reagents and solvents were purchased from commercial suppliers and used without further purification.

\section{General procedure for the Grignard reaction}

Analogously as described in [23], Mg turnings and iodine in THF were heated to reflux until the brown colour disappeared. Then, 5-bromo-1-pentene (273 mg, $1.92 \mathrm{mmol}$ ) was added and the reaction mixture was stirred for $30 \mathrm{~min}$. Next, thiophene 2,5-dialdehyde $(4,100 \mathrm{mg}, 0.71 \mathrm{mmol})$ was added and the resulting mixture was stirred and heated at reflux for $3 \mathrm{~h}$. After completion of the reaction (TLC monitoring), $2 \mathrm{~N} \mathrm{HCl}$ was added and reaction mixture was stirred for $30 \mathrm{~min}$. The reaction mixture was diluted with EtOAc $(10 \mathrm{~mL})$ and $\mathrm{H}_{2} \mathrm{O}(10 \mathrm{~mL})$ and extracted with EtOAc. The organic layer was washed with brine, dried over $\mathrm{Na}_{2} \mathrm{SO}_{4}$, and concentrated under reduced pressure. The crude products were purified by column chromatography to obtain the diol $\mathbf{6}$ (or $\mathbf{6 a}$ ).

Compound 6: Semi solid, $104 \mathrm{mg}$ (52\%), by using the general procedure $100 \mathrm{mg}(0.71 \mathrm{mmol})$ of thiophene-2,5-carbaldehyde 4 was reacted with 4-pentenylmagnesium bromide. IR (neat): 3943, 3677, 3601, 3050, 2923, 1261, $739 \mathrm{~cm}^{-1}$; ${ }^{1} \mathrm{H}$ NMR $\left(400 \mathrm{MHz}, \mathrm{CDCl}_{3}\right) \delta 1.35-1.48(\mathrm{~m}, 2 \mathrm{H}), 1.50-1.60(\mathrm{~m}, 2 \mathrm{H})$, $1.74-1.89$ (m, 4H), 2.09 (q, $J=7.10 \mathrm{~Hz}, 4 \mathrm{H}), 2.59$ (bs, 2H), $4.81(\mathrm{t}, J=6.50 \mathrm{~Hz}, 2 \mathrm{H}), 4.94-5.03(\mathrm{~m}, 4 \mathrm{H}), 5.73-5.83(\mathrm{~m}$, $2 \mathrm{H}), 6.78$ (s, 2H) ppm; ${ }^{13} \mathrm{C} \mathrm{NMR}\left(100.6 \mathrm{MHz}, \mathrm{CDCl}_{3}\right) \delta 25.15$, 33.53, 38.60, 70.36, 70.39, 114.93, 123.33, 138.57, 147.99; HRMS (Q-Tof) $m / z$ : $[\mathrm{M}+\mathrm{Na}]^{+}$calcd for $\mathrm{C}_{16} \mathrm{H}_{24} \mathrm{NaO}_{2} \mathrm{~S}$, 303.1389; found, 303.1394 .

Compound 6a: Semi solid, $107 \mathrm{mg}$ (48\%), by using the general procedure $100 \mathrm{mg}(0.71 \mathrm{mmol})$ of thiophene-2,5-carbaldehyde 4 was reacted with 5-hexenylmagnesium bromide. IR (neat): 743, 1270, 2933, 3042, 3589, 3694, $3942 \mathrm{~cm}^{-1}$; ${ }^{1} \mathrm{H}$ NMR $\left(400 \mathrm{MHz}, \mathrm{CDCl}_{3}\right) \delta 1.27-1.52(\mathrm{~m}, 8 \mathrm{H}), 1.72-1.89(\mathrm{~m}, 4 \mathrm{H})$, 2.01-2.11 (m, 4H), 2.57 (bs, 2H), 4.77-4.84 (m, 2H), 4.91-5.03 $(\mathrm{m}, 4 \mathrm{H}), 5.73-5.83(\mathrm{~m}, 2 \mathrm{H}), 6.77(\mathrm{~s}, 2 \mathrm{H}) \mathrm{ppm} ;{ }^{13} \mathrm{C} \mathrm{NMR}$ $\left(100.6 \mathrm{MHz}, \mathrm{CDCl}_{3}\right) \delta 25.41,28.74,33.73,39.03,70.43$, $114.58,123.33,138.89,148.03$ ppm; HRMS (Q-Tof) $m / z$ : $[\mathrm{M}+\mathrm{H}]^{+}$calcd for $\mathrm{C}_{18} \mathrm{H}_{29} \mathrm{O}_{2} \mathrm{~S}, 309.1888$; found, 309.1959.

\section{General procedure for the $\mathrm{MnO}_{2}$ oxidation}

To the solution of diol derivative 6 (or $\mathbf{6 a})\left(50 \mathrm{mg}\right.$ ) in $\mathrm{CH}_{2} \mathrm{Cl}_{2}$ $\left(10 \mathrm{~mL}\right.$ ) was added $\mathrm{MnO}_{2}$ (4 equiv) as the oxidizing agent at $\mathrm{rt}$ and reaction mixture was heated at reflux overnight. After completion of the reaction (TLC monitoring), the crude reaction mixture was filtered through a Celite pad (washed with $\mathrm{CH}_{2} \mathrm{Cl}_{2}$ ) and concentrated under reduced pressure. The crude product was purified by column chromatography (silica gel; $5 \%$ EtOAc/petroleum ether) to give bisalkene dione derivative $\mathbf{3}$ (or 3a).

Compound 3: Semi solid, $71 \mathrm{mg}$ (73\%), by using the general procedure $100 \mathrm{mg}(0.35 \mathrm{mmol})$ of thiophene derivative 6 was oxidized with $\mathrm{MnO}_{2}$ to deliver 3. IR (neat): 738, 1267, 1687, 2934, 3055, 3357, 3690, $3945 \mathrm{~cm}^{-1}$; ${ }^{1} \mathrm{H}$ NMR (400 MHz, $\left.\mathrm{CDCl}_{3}\right) \delta 1.84(\mathrm{q}, J=7.28 \mathrm{~Hz}, 4 \mathrm{H}), 2.15(\mathrm{q}, J=7.05 \mathrm{~Hz}, 4 \mathrm{H})$, $2.93(\mathrm{t}, J=4.12 \mathrm{~Hz}, 4 \mathrm{H}), 4.99-5.07(\mathrm{~m}, 4 \mathrm{H}), 5.75-5.85(\mathrm{~m}$, 2H), 7.67 (s, 2H) ppm; ${ }^{13} \mathrm{C} \mathrm{NMR}\left(100.6 \mathrm{MHz}, \mathrm{CDCl}_{3}\right) \delta 23.45$, 33.16, 38.86, 115.77, 131.52, 137.81, 148.82, 193.55 ppm; HRMS (Q-Tof) m/z: $[\mathrm{M}+\mathrm{H}]^{+}$calcd for $\mathrm{C}_{16} \mathrm{H}_{21} \mathrm{O}_{2} \mathrm{~S}, 277.1262$; found, 277.1266 .

Compound 3a: Semi solid, $74 \mathrm{mg}$ (75\%), by using the general procedure $100 \mathrm{mg}(0.32 \mathrm{mmol})$ of thiophene derivative $6 \mathbf{a}$ was oxidized with $\mathrm{MnO}_{2}$ to deliver 3a. IR (neat): 740, 1270, 1685, 2939, 3051, 3361, 3689, $3950 \mathrm{~cm}^{-1}$; ${ }^{1} \mathrm{H}$ NMR (400 MHz, $\left.\mathrm{CDCl}_{3}\right): \delta=1.44-1.49(\mathrm{~m}, 4 \mathrm{H}), 1.73-1.80(\mathrm{~m}, 4 \mathrm{H}), 2.10(\mathrm{q}, J=$ $7.24 \mathrm{~Hz}, 4 \mathrm{H}), 2.92(\mathrm{t}, J=7.50 \mathrm{~Hz}, 4 \mathrm{H}), 4.95-5.05(\mathrm{~m}, 4 \mathrm{H})$, 5.75-5.85 (m, 2H), 7.67 (s, 2H) ppm; ${ }^{13} \mathrm{C} \mathrm{NMR} \mathrm{(100.6} \mathrm{MHz,}$ $\left.\mathrm{CDCl}_{3}\right) \delta 24.05,28.59,33.66,39.68,115.03,131.55,138.50$, 148.83, 193.68 ppm; HRMS (Q-Tof) $m / z:[\mathrm{M}+\mathrm{H}]^{+}$calcd for $\mathrm{C}_{18} \mathrm{H}_{25} \mathrm{O}_{2} \mathrm{~S}, 305.1574$; found, 305.1557 . 


\section{General procedure for the preparation of diindole derivatives}

Analogously as described in $[39,40]$, in a typical experiment, $1.5 \mathrm{~g}$ of a mixture of $\mathrm{L}-(+)$-tartaric acid $/ N, N^{\prime}$-dimethylurea (30:70) was heated to $70{ }^{\circ} \mathrm{C}$ to obtain a clear melt. To this melt, $2 \mathrm{mmol}$ of $\mathrm{N}$-methyl- $\mathrm{N}$-phenylhydrazine and $1 \mathrm{mmol}$ of diketone were added at $70{ }^{\circ} \mathrm{C}$. After completion of the reaction (TLC monitoring by mini work up), the reaction mixture was quenched with water while it was still hot. The reaction mixture was cooled to $\mathrm{rt}$ and the solid was filtered through a sintered glass funnel and washed with water $(2 \times 5 \mathrm{~mL})$. The crude product was dried under vacuum and then it was purified by silica gel column chromatography.

Compound 5: Pale yellow oil, $123 \mathrm{mg}$ (75\%), by using the general procedure $100 \mathrm{mg}(0.36 \mathrm{mmol})$ of dione 3 was converted into diindole derivative 5. IR (neat): 1048, 1097, 1242, 1374, 1447, 1465, 2927, 2974, $3019 \mathrm{~cm}^{-1}$; ${ }^{1} \mathrm{H}$ NMR $\left(500 \mathrm{MHz}, \mathrm{CDCl}_{3}\right) \delta 2.49-2.51(\mathrm{~m}, 4 \mathrm{H}), 2.99-3.04(\mathrm{~m}, 4 \mathrm{H})$, $3.80(\mathrm{~s}, 6 \mathrm{H}), 5.00-5.13(\mathrm{~m}, 4 \mathrm{H}), 5.92-5.98(\mathrm{~m}, 2 \mathrm{H}), 7.20-7.24$ $(\mathrm{m}, 4 \mathrm{H}), 7.32-7.36(\mathrm{~m}, 2 \mathrm{H}), 7.39-7.41(\mathrm{~m}, 2 \mathrm{H}), 7.70-7.73$ $(\mathrm{m}, 2 \mathrm{H}) \mathrm{ppm} ;{ }^{13} \mathrm{C} \mathrm{NMR}\left(100.6 \mathrm{MHz}, \mathrm{CDCl}_{3}\right) \delta 24.86$, $31.06,35.70,109.65,114.83,115.88,119.44,119.53,122.59$, 127.52, 129.16, 129.72, 134.45, 137.67, 138.79 ppm; HRMS (Q-Tof) $m / z$ : $[\mathrm{M}+\mathrm{H}]^{+}$calcd for $\mathrm{C}_{30} \mathrm{H}_{31} \mathrm{~N}_{2} \mathrm{~S}, 451.2208$; found, 451.2212 .

Compound 5a: Pale yellow oil, $110 \mathrm{mg}$ (70\%), by using the general procedure $100 \mathrm{mg}(0.33 \mathrm{mmol})$ of dione 3a was converted into bisindole derivative 5a. IR (neat): 738, 1267, 2934, 3055, 3357, 3690, $3945 \mathrm{~cm}^{-1}$; ${ }^{1} \mathrm{H}$ NMR (500 MHz, $\left.\mathrm{CDCl}_{3}\right) \delta 1.83(\mathrm{t}, J=6.50 \mathrm{~Hz}, 4 \mathrm{H}), 2.15-2.16(\mathrm{~m}, 4 \mathrm{H})$, 2.89-2.93 (m, 4H), $3.78(\mathrm{~s}, 6 \mathrm{H}), 4.95-5.04(\mathrm{~m}, 4 \mathrm{H}), 5.81-5.90$ $(\mathrm{m}, 2 \mathrm{H}), 7.18-7.21(\mathrm{~m}, 4 \mathrm{H}), 7.30-7.33(\mathrm{~m}, 2 \mathrm{H}), 7.37-7.39(\mathrm{~m}$, 2H), 7.67-7.79 (m, 2H) ppm; ${ }^{13} \mathrm{C}$ NMR (125.6 MHz, $\left.\mathrm{CDCl}_{3}\right) \delta$ 24.59, 30.69, 31.04, 33.91, 109.63, 114.67, 116.52, 119.48, 122.57, 127.62, 129.18, 129.67, 134.56, 137.70, $138.92 \mathrm{ppm}$; HRMS (Q-Tof) $m / z$ : $[\mathrm{M}+\mathrm{H}]^{+}$calcd for $\mathrm{C}_{32} \mathrm{H}_{35} \mathrm{~N}_{2} \mathrm{~S}, 479.2521$; found, 479.2548 .

\section{General procedure for RCM reaction}

Analogously as described in [42], a solution of bisindole-alkene derivative $5(0.05 \mathrm{mmol})$ in dry $\mathrm{CH}_{2} \mathrm{Cl}_{2}(50 \mathrm{~mL})$ was degassed with $\mathrm{N}_{2}$ gas for $10 \mathrm{~min}$. Then, Grubbs' second generation catalyst $(10 \mathrm{~mol} \%)$ was added and the reaction mixture was stirred at room temperature for $24 \mathrm{~h}$. After completion of the reaction (TLC monitoring), the solvent was removed under reduced pressure and the crude product was purified by silica gel column chromatography (5\% EtOAc/ petroleum ether) to give the RCM compound $\mathbf{1}$ as a colourless solid.
Compound 1: White solid, $25 \mathrm{mg}(90 \%)$, by using the general procedure $30 \mathrm{mg}(0.06 \mathrm{mmol})$ of bisindole 5 was treated with Grubbs' second generation catalyst to deliver RCM product $\mathbf{1}$. Mp 187-189 ${ }^{\circ} \mathrm{C}$; IR (neat): 1098, 1265, 1364, 1458, 1644, 1734, 2858, $2926 \mathrm{~cm}^{-1}$; ${ }^{1} \mathrm{H}$ NMR $\left(400 \mathrm{MHz}, \mathrm{CDCl}_{3}\right) \delta$ 2.09-2.15 (m, 4H), 2.96-3.01 (m, 4H), $3.92(\mathrm{~s}, 6 \mathrm{H}), 5.63(\mathrm{t}, J=$ $5.40 \mathrm{~Hz}, 2 \mathrm{H}), 7.15-7.19(\mathrm{~m}, 4 \mathrm{H}), 7.28-7.30$ (m, 2H), 7.37 (d, $J$ $=8.16 \mathrm{~Hz}, 2 \mathrm{H}), 7.65(\mathrm{~d}, J=7.88 \mathrm{~Hz}, 2 \mathrm{H}) \mathrm{ppm} ;{ }^{13} \mathrm{C} \mathrm{NMR}$ $\left(125.6 \mathrm{MHz}, \mathrm{CDCl}_{3}\right): \delta 26.20,28.14,30.80,109.64,115.73$, $118.89,119.65,122.54,127.75,128.23,130.16,130.31,134.28$, $137.05 \mathrm{ppm}$; HRMS (Q-Tof) $m / z:[\mathrm{M}+\mathrm{H}]^{+}$calcd for $\mathrm{C}_{28} \mathrm{H}_{27} \mathrm{~N}_{2} \mathrm{~S}$, 423.1895; found, 423.1893 .

Compound 1a: White solid, $35 \mathrm{mg}$ (93\%), By using the general procedure $40 \mathrm{mg}(0.08 \mathrm{mmol})$ of diindole $\mathbf{5 a}$ was treated with Grubbs' second generation catalyst to deliver RCM product 1a. Mp $183-185^{\circ} \mathrm{C}$; IR (neat): 1048, 1245, 1374, 1448, 1742, 1889, 2085, 2943, 2987, 3464, $3628 \mathrm{~cm}^{-1}$; ${ }^{1} \mathrm{H}$ NMR (500 MHz, $\left.\mathrm{CDCl}_{3}\right) \delta 1.76-1.78(\mathrm{~m}, 4 \mathrm{H}), 2.03(\mathrm{~d}, J=5.25 \mathrm{~Hz}, 4 \mathrm{H}), 2.96(\mathrm{t}$, $J=7.80 \mathrm{~Hz}, 4 \mathrm{H}), 3.80(\mathrm{~s}, 6 \mathrm{H}), 5.37(\mathrm{~s}, 2 \mathrm{H}), 7.13-7.18(\mathrm{~m}, 4 \mathrm{H})$, $7.27-7.30(\mathrm{~m}, 2 \mathrm{H}), 7.35-7.37(\mathrm{~m}, 2 \mathrm{H}), 7.67(\mathrm{~d}, J=7.85 \mathrm{~Hz}$, $2 \mathrm{H}) \mathrm{ppm} ;{ }^{13} \mathrm{C} \mathrm{NMR}\left(125.6 \mathrm{MHz}, \mathrm{CDCl}_{3}\right) \delta 23.33,30.45$, 30.96, 31.14, 109.64, 116.79, 119.43, 122.49, 127.68, 128.95, 129.68, 130.52, 134.11, 137.58 ppm; HRMS (Q-Tof) $\mathrm{m} / z$ : $[\mathrm{M}+\mathrm{H}]^{+}$calcd for $\mathrm{C}_{30} \mathrm{H}_{31} \mathrm{~N}_{2} \mathrm{~S}, 451.2208$; found, 451.2192.

\section{Supporting Information}

\section{Supporting Information File 1}

Copies of ${ }^{1} \mathrm{H},{ }^{13} \mathrm{C}$ NMR and HRMS spectra for all new compounds.

[http://www.beilstein-journals.org/bjoc/content/ supplementary/1860-5397-11-165-S1.pdf]

\section{Acknowledgements}

We thank the Department of Science and Technology (DST), New Delhi for the financial support and Sophisticated Analytical Instrument Facility (SAIF), IIT-Bombay for recording spectral data. S. K. thanks the Department of Science and Technology for the award of a J. C. Bose fellowship. A. K. C. thanks the University Grants Commission, New Delhi for the award of a research fellowship. M. E. S. thanks the IIT-Bombay for the award of a research fellowship.

\section{References}

1. Nilewski, C.; Carreira, E. M. Eur. J. Org. Chem. 2012, 1685-1698. doi:10.1002/ejoc.201101525

2. Hopf, H.; Gleiter, R. Modern Cyclophane Chemistry; Wiley-VCH: Weinheim, Germany, 2004. 
3. Keehn, P. M.; Rosenfeld, S. M. Cyclophanes; Acadamic Press: New York, 1983; Vol. 2.

4. Pigge, F. C.; Ghasedi, F.; Rath, N. P. J. Org. Chem. 2002, 67, 4547-4552. doi:10.1021/jo0256181

5. Gibe, R.; Green, J. R.; Davidson, G. Org. Lett. 2003, 5, 1003-1005 doi:10.1021/ol027564n

6. Reiser, O.; König, K.; Meerholz, K.; Heinze, J.; Wellauer, T.; Gerson, F.; Frim, R.; Rabinovitz, M.; de Meijere, A. J. Am. Chem. Soc. 1993, 115, 3511-3518. doi:10.1021/ja00062a015

7. Fallis, A. G. Synthesis 2004, 2249-2267. doi:10.1055/s-2004-832847

8. Frampton, M. J.; Anderson, H. L. Angew. Chem., Int. Ed. 2007, 46, 1028-1064. doi:10.1002/anie.200601780

9. Xi, H.-T.; Zhao, T.; Sun, X.-Q.; Miao, C.-B.; Zong, T.; Meng, Q. RSC Adv. 2013, 3, 691-694. doi:10.1039/c2ra22802e

10. Wex, B.; Jradi, F. M.; Patra, D.; Kaafarani, B. R. Tetrahedron 2010, 66, 8778-8784. doi:10.1016/j.tet.2010.08.073

11. Kotha, S. Acc. Chem. Res. 2003, 36, 342-351. doi:10.1021/ar020147q

12. Fürstner, A.; Stelzer, F.; Rumbo, A.; Krause, H. Chem. - Eur. J. 2002, 8, 1856-1871. doi:10.1002/1521-3765(20020415)8:8<1856::AID-CHEM1856>3.0.CO; 2-R

13. Huang, M.; Song, L.; Liu, B. Org. Lett. 2010, 12, 2504-2507. doi:10.1021/ol100692x

14. Kotha, S.; Chavan, A. S.; Shaikh, M. J. Org. Chem. 2012, 77, 482-489. doi:10.1021/jo2020714

15. Alcaide, B.; Almendros, P.; Quirós, M. T.; Lázaro, C.; Torres, M. R. J. Org. Chem. 2014, 79, 6244-6255. doi:10.1021/jo500993x

16. Kotha, S.; Mandal, K. Chem. - Asian J. 2009, 4, 354-362. doi:10.1002/asia.200800244

17. Kotha, S.; Dipak, M. K. Tetrahedron 2012, 68, 397-421. doi:10.1016/j.tet.2011.10.018

18. Kotha, S.; Sreenivasachary, N.; Mohanraja, K.; Durani, S. Bioorg. Med. Chem. Lett. 2001, 11, 1421-1423. doi:10.1016/S0960-894X(01)00227-X

19. Kotha, S.; Sreenivasachary, N. Bioorg. Med. Chem. Lett. 1998, 8, 257-260. doi:10.1016/S0960-894X(98)00002-X

20. Kotha, S.; Bansal, D.; Singh, K.; Banerjee, S. J. Organomet. Chem. 2011, 696, 1856-1860. doi:10.1016/j.jorganchem.2011.02.019

21. Kotha, S.; Manivannan, E. ARKIVOC 2003, No. iii, 67-76.

22. Kotha, S.; Ali, R.; Chinnam, A. K. Tetrahedron Lett. 2014, 55, 4492-4495. doi:10.1016/j.tetlet.2014.06.049

23. Kotha, S.; Waghule, G. T.; Shirbhate, M. E. Eur. J. Org. Chem. 2014, 984-992. doi:10.1002/ejoc.201301493

24. Kotha, S.; Shirbhate, M. E. Tetrahedron Lett. 2014, 55, 6972-6975. doi:10.1016/j.tetlet.2014.10.092

25. Kotha, S.; Waghule, G. T. Tetrahedron Lett. 2014, 55, 4264-4268. doi:10.1016/j.tetlet.2014.05.129

26. Raatikainen, K.; Huuskonen, J.; Kolehmainen, E.; Rissanen, K. Chem. - Eur. J. 2008, 14, 3297-3305. doi:10.1002/chem.200701862

27. Rajakumar, P.; Swaroop, M. G. Tetrahedron Lett. 2006, 47, 3019-3022. doi:10.1016/j.tetlet.2006.03.013

28. Dohm, J.; Vögtle, F. Top. Curr. Chem. 1992, 161, 69-106. doi:10.1007/3-540-54348-1_8

29. Matsuoka, Y.; Ishida, Y.; Sasaki, D.; Saigo, K. Chem. - Eur. J. 2008, 14, 9215-9222. doi:10.1002/chem.200800942

30. Tanaka, K. Synlett 2007, 1977-1993. doi:10.1055/s-2007-984541

31. Garrison, J. C.; Panzner, M. J.; Tessier, C. A.; Youngs, W. J. Synlett 2005, 99-102. doi:10.1055/s-2004-836042

32. Deslongchamps, P. Aldrichimica Acta 1991, 24, 43-56.
33. Mikhaleva, A. I.; Ivanov, A. V.; Skital'tseva, E. V.; Ushakov, I. A.; Vasil'tsov, A. M.; Trofimov, B. A. Synthesis 2009, 587-590. doi:10.1055/s-0028-1083312

34. Wei, X.; Taylor, R. J. K. J. Org. Chem. 2000, 65, 616-620. doi:10.1021/jo9913558

35. Shon, Y.-S.; Lee, T. R. Tetrahedron Lett. 1997, 38, 1283-1286. doi:10.1016/S0040-4039(97)00072-5

36. Ghosh, S.; Ghosh, S.; Sarkar, N. J. Chem. Sci. 2006, 118, 223-235.

37. Samojłowicz, C.; Grela, K. ARKIVOC 2011, No. iv, 71-81.

38. Gore, S.; Baskaran, S.; König, B. Org. Lett. 2012, 14, 4568-4571. doi:10.1021/ol302034r

39. Kotha, S.; Chinnam, A. K. Synthesis 2014, 46, 301-306. doi:10.1055/s-0033-1340341

40. Kotha, S.; Chinnam, A. K. Heterocycles 2015, 90, 690-697. doi:10.3987/COM-14-S(K)35

41. CCDC 1060941 contains the supplementary crystallographic data for this paper. These data can be obtained free of charge from The Cambridge Crystallographic Data Centre via http://www.ccdc.cam.ac.uk

42. Kotha, S.; Chavan, A. S.; Dipak, M. K. Tetrahedron 2011, 67, 501-504 doi:10.1016/j.tet.2010.10.080

\section{License and Terms}

This is an Open Access article under the terms of the Creative Commons Attribution License (http://creativecommons.org/licenses/by/2.0), which permits unrestricted use, distribution, and reproduction in any medium, provided the original work is properly cited.

The license is subject to the Beilstein Journal of Organic Chemistry terms and conditions:

(http://www.beilstein-journals.org/bjoc)

The definitive version of this article is the electronic one which can be found at: doi:10.3762/bjoc. 11.165 